\title{
Similarity reduction and closed form solutions for a model derived from two-layer fluids
}

\author{
Marianna Ruggieri ${ }^{*}$ and Maria Paola Speciale ${ }^{2}$
}

\section{"Correspondence:}

marianna.ruggieri@unikore.it

${ }^{1}$ Faculty of Engineering and

Architecture, Kore University of

Enna, Enna, Italy

Full list of author information is

available at the end of the article

\begin{abstract}
In this paper, we study an integrable system of coupled KdV equations, derived by Gear and Grimshaw (Stud. Appl. Math. 70(3):235-258, 1984), modeling the strong interaction of two-dimensional, long, internal gravity waves propagating on neighboring pycnoclines in a stratified fluid. In particular, we present the complete group classification of the model and find conditions on arbitrary parameters for which the system admits symmetries. Some exact solutions of physical relevance are derived.
\end{abstract}

\section{Introduction}

In the last years, some types of coupled Korteweg-de Vries (KdV) equations have been encountered in various natural science fields and they are relevant in physical systems such as plasmas, fluids and for describing the lattice vibrations of a crystal at low temperatures. In the literature, some kinds of coupled KdV equations have been introduced to describe two resonantly interacting normal modes of internal-gravity waves in a shallow stratified liquid [1].

Having in mind the rich treasure of nonlinear coupled KdV equations, in this paper we turn our attention to a system introduced by Gear and Grimshaw in [1] to model interactions of solitary waves in a stratified fluid. The model under consideration has the structure of a pair of Korteweg-de Vries equations coupled through both dispersive and nonlinear terms:

$$
\begin{aligned}
& u_{t}+u_{x x x}+2 u u_{x}+2 e_{1} v v_{x}+e_{2}\left(u_{x} v+u v_{x}\right)+e_{3} v_{x x x}=0, \\
& c_{1} v_{t}+v_{x x x}+2 v v_{x}+c_{2} v_{x}+c_{3}\left[e_{1}\left(u_{x} v+u v_{x}\right)+2 e_{2} u u_{x}+e_{3} u_{x x x}\right]=0,
\end{aligned}
$$

where $u(t, x)$ and $v(t, x)$ are the dependent variables and subscripts denote partial derivative with respect to the independent variables $t$ and $x$; moreover, $e_{i}(i=1,2,3)$ and $c_{j}$ $(j=1,2,3)$ represent arbitrary constants.

Some mathematical questions related to the Cauchy problem (CP) of (1) were studied by Bona et al. [2] who showed that the (CP) is globally well posed in suitably strong function spaces. Moreover, Bona and Saut also stated that system (1) is susceptible of experiencing the dispersive blow-up phenomenon. Following the guide of the approximate method

@2013Ruggieri and Speciale; licensee Springer. This is an Open Access article distributed under the terms of the Creative Commons Attribution License (http://creativecommons.org/licenses/by/2.0), which permits unrestricted use, distribution, and reproduction in any medium, provided the original work is properly cited. 
proposed in [3] (see references therein for a review), an approximate analysis of model (1) is performed in [4].

Motivated by a great number of physical applications, we focus our attention on Lie symmetries admitted by system (1) in order to find conditions on real parameters for which the system does admit symmetries. This is done because the group classification problem is interesting not only from a purely mathematical point of view, but is also important in the applications [5-7]. Originally developed by Lie at the beginning of the nineteenth century, the symmetry analysis plays a key role in almost all scientific fields.

This paper is arranged as follows. In Section 2, we briefly explain the basic definitions and notations required to perform the Lie group analysis, and we provide the vector fields of the symmetries admitted by coupled KdV equations (1). In Section 3, we use some symmetries to reduce (1) and find some exact solutions, whose snapshots are provided in order to show their properties.

\section{Symmetry group of coupled KdV equations}

In order to discuss the group classification of system (1), we apply the classical Lie theory. Symmetry analysis is very successfully used in several branches of sciences and plays an important role in the theory of differential equations since it allows for the development of systematic procedures to integrate by quadrature - or at least lower the order - of ODEs; in the case of PDEs, Lie symmetries may lead to the determination of invariant solutions of a variety of initial and boundary value problems in different fields such as transport, reaction and diffusion, dissipative phenomena [8-14], of unsteady solutions starting from known steady solutions when the equations are invariant with respect to the so-called projective group (for instance, two-dimensional Euler equations) [15], or to the construction of relations between different differential equations that turn out to be equivalent [16-20]; moreover, by using symmetries of classes of differential equations (equivalence transformations [21,22]) it is possible to map a system of balance laws to an equivalent system of conservation laws $[23,24]$.

Then, we look for the one-parameter Lie group of infinitesimal transformations in $(t, x, u, v)$-space given by

$$
\begin{aligned}
& \hat{t}=t+\varepsilon \xi^{1}(t, x, u, v)+\mathcal{O}\left(\varepsilon^{2}\right), \\
& \hat{x}=x+\varepsilon \xi^{2}(t, x, u, v)+\mathcal{O}\left(\varepsilon^{2}\right), \\
& \hat{u}=u+\varepsilon \eta^{1}(t, x, u, v)+\mathcal{O}\left(\varepsilon^{2}\right), \\
& \hat{v}=v+\varepsilon \eta^{2}(t, x, u, v)+\mathcal{O}\left(\varepsilon^{2}\right),
\end{aligned}
$$

where $\varepsilon$ is the group parameter and the associated Lie algebra $\mathcal{L}$ is the set of vector fields of the form

$$
X=\xi^{1} \frac{\partial}{\partial t}+\xi^{2} \frac{\partial}{\partial x}+\eta^{1} \frac{\partial}{\partial u}+\eta^{2} \frac{\partial}{\partial v}
$$

We then require the invariance conditions (see [6, 7]), considering the third prolongation of the operator $X$, under the constraints that the equations at hand be satisfied. The determining system leads to the following results: for arbitrary values of constants, $e_{i}(i=1,2,3)$ 
and $c_{j}(j=1,2,3)$, we have that the principal Lie algebra $\mathcal{L}_{P}$ of system (1) is two-dimensional and is spanned by the operators

$$
X_{1}=\frac{\partial}{\partial t}, \quad X_{2}=\frac{\partial}{\partial x} .
$$

On the contrary, the complete symmetry classification of model (1) gives the following extensions of the principal Lie algebra.

Case 1.

If one of the parameters $c_{3}, e_{1}$ or $e_{2}$ is zero, we are able to write in a unified form the corresponding operator

$$
X_{3}=3 k t \frac{\partial}{\partial t}+\left[k x-2 c_{2}\left(e_{2}^{2}-4 e_{1}\right) t\right] \frac{\partial}{\partial x}-2\left(k u-2 e_{1} c_{2}\right) \frac{\partial}{\partial u}-2\left(k v+e_{2} c_{2}\right) \frac{\partial}{\partial v},
$$

with the position $k=2\left(e_{2}-c_{3} e_{1}^{2}\right)-c_{1}\left(e_{2}^{2}-4 e_{1}\right)$.

A special case arises when both $k$ and $c_{2}$ are equal to zero; in such a situation, we have operators

$$
\begin{aligned}
& X_{3}=3 t \frac{\partial}{\partial t}+x \frac{\partial}{\partial x}-2 u \frac{\partial}{\partial u}-2 v \frac{\partial}{\partial v}, \\
& \dot{X}_{4}=-\left(e_{2}^{2}-4 e_{1}\right) t \frac{\partial}{\partial x}+2 e_{1} \frac{\partial}{\partial u}-e_{2} \frac{\partial}{\partial v} .
\end{aligned}
$$

Case 2.

When $c_{1}=1$ and $e_{3}=0$, we get $c_{3}=\frac{e_{2}^{2}-e_{2}-2-4 e_{1}}{e_{1}\left(2 e_{1}+e_{2}\right)}\left(e_{2} \neq-2 e_{1}, e_{1} \neq 0\right)$ along with the following constraint on parameters:

$$
e_{2}^{3}-\left(2+e_{1}\right) e_{2}^{2}+4 e_{1} e_{2}+\left(4 e_{1}+1\right) e_{1}=0 .
$$

We get the operator

$$
\begin{aligned}
\tilde{X}_{3}= & 3 e_{2} t \frac{\partial}{\partial t}+\left(\frac{2 k_{0} c_{2} t}{\Delta}+e_{2} x\right) \frac{\partial}{\partial x} \\
& +2\left[\frac{c_{2} e_{1} k_{1}}{\Delta}+\frac{3 e_{1} e_{2} k_{2}}{\Delta} v-e_{2}\left(\frac{3 e_{2} k_{3}}{\Delta}+1\right) u\right] \frac{\partial}{\partial u} \\
& +2 e_{2}\left[\frac{c_{2} k_{4}}{\Delta}+\frac{3 k_{5}}{\Delta} u+\left(\frac{3 e_{1} k_{3}}{\Delta}-1\right) v\right] \frac{\partial}{\partial v},
\end{aligned}
$$

with $\Delta$ and $k_{i}=k_{i}\left(e_{1}, e_{2}\right)(i=1, \ldots, 5)$ given by

$$
\begin{aligned}
& k_{0}=\left(2 e_{1}+e_{2}\right)\left(24 e_{1}^{2}-18 e_{1} e_{2}^{2}+10 e_{1} e_{2}+8 e_{1}+3 e_{2}^{4}-4 e_{2}^{3}-5 e_{2}^{2}\right), \\
& k_{1}=\left(2 e_{1}+e_{2}\right)\left(12 e_{1}-3 e_{2}^{2}+5 e_{2}+4\right), \\
& k_{2}=\left(2 e_{1}+e_{2}\right)\left(e_{2}^{2}-e_{2}-4 e_{1}-2\right), \\
& k_{3}=\left(1-e_{2}\right)\left(e_{2}^{2}-e_{2}-4 e_{1}-2\right), \\
& k_{4}=\left(2 e_{1}+e_{2}\right)\left(3 e_{2}^{2}-4 e_{2}-12 e_{1}-5\right), \\
& k_{5}=\left(e_{2}^{2}-2 e_{2}-4 e_{1}-1\right)\left(e_{2}^{2}-e_{2}-4 e_{1}-2\right), \\
& \Delta=3\left(e_{1}-e_{2}\right)\left(e_{2}-1\right)\left(e_{2}^{2}-e_{2}-4 e_{1}-2\right),
\end{aligned}
$$


where $\Delta \neq 0$; on the contrary, if $\Delta=0$, we fall in other cases of the classification (if $e_{1}=e_{2}$, we fall in Case 4 , if $e_{2}=1$ or $e_{2}^{2}-e_{2}-4 e_{1}-2=0$, we recover Case 3 ).

Case 3.

A singularity arises when $e_{2}=-2 e_{1}$, that leads us to considering the special values $e_{2}=1$, $e_{1}=-\frac{1}{2}, c_{3}=2(5 \pm 3 \sqrt{3})$; then the related operator reads

$$
\begin{aligned}
\bar{X}_{3}= & 3 \bar{k} t \frac{\partial}{\partial t}+\left[\bar{k} x-24 c_{2}\left(5 c_{3}+4\right) t\right] \frac{\partial}{\partial x} \\
& -2\left(2 c_{2}\left(8+7 c_{3}\right)+\left(\bar{k}-6 c_{3}\left(c_{3}-4\right)\right) u-18 c_{3} v\right) \frac{\partial}{\partial u} \\
& +2\left(-16 c_{2}\left(1+2 c_{3}\right)+12\left(c_{3}+2\right) c_{3} u-\left(\bar{k} v-3 c_{3}\left(c_{3}-4\right)\right) v\right) \frac{\partial}{\partial v},
\end{aligned}
$$

where $\bar{k}=-7 c_{3}^{2}-4 c_{3}-16$.

Case 4

Finally, another singularity arises if $e_{1}=0$ and $e_{2}=0$, whereupon we obtain

$$
\hat{X}_{3}=3 t \frac{\partial}{\partial t}+x \frac{\partial}{\partial x}-2 u \frac{\partial}{\partial u}-\left(c_{2}+2 v\right) \frac{\partial}{\partial v},
$$

and

$$
\hat{X}_{4}=2 t \frac{\partial}{\partial x}+\frac{\partial}{\partial u}+c_{1} \frac{\partial}{\partial v} .
$$

\section{Exact solutions}

In this section, we construct some exact solutions which are invariant with respect to some of the symmetries determined above. The main advantage of the procedure is that it allows us to find solutions of the original system of partial differential equations (PDEs) by solving a system of reduced equations which are ordinary differential equations.

Starting from the operator $X_{1}+a_{2} X_{2}+a_{4} \hat{X}_{4}$, we obtain the following similarity variable and similarity solution:

$$
\begin{aligned}
& z=x-a_{2} t-a_{4} t^{2}, \\
& u(x, t)=a_{4} t+U(z), \quad v(x, t)=c_{1} a_{4} t+V(z) .
\end{aligned}
$$

The reduced functions $U(z)$ and $V(z)$ must satisfy the ODEs

$$
\begin{aligned}
& e_{3} V^{\prime \prime \prime}+U^{\prime \prime \prime}+U^{\prime}\left(2 U-a_{2}\right)-a_{4}=0, \\
& V^{\prime \prime \prime}+c_{3} e_{3} U^{\prime \prime \prime}+V^{\prime}\left(2 V+c_{2}-a_{2} c_{1}\right)+a_{4} c_{1}^{2}=0 .
\end{aligned}
$$

A particular solution of (14) is

$$
\begin{aligned}
& U(z)=\frac{1}{2}\left(a_{2} \pm \sqrt{a_{2}^{2}-4 a_{4} z-4 \alpha_{7}}\right), \\
& V(z)=-\frac{1}{2 e_{3}}\left(a_{2} \pm \sqrt{a_{2}^{2}-4 a_{4} z-4 \alpha_{7}}\right) .
\end{aligned}
$$


Then the corresponding solution of (1) reads as

$$
\begin{aligned}
& u(x, t)=a_{4} t+\frac{1}{2}\left[a_{2} \pm \sqrt{a_{2}^{2}-4 a_{4}\left(x-a_{2} t-a_{4} t^{2}\right)-4 \alpha_{7}}\right], \\
& v(x, t)=c_{1} a_{4} t-\frac{1}{2 e_{3}}\left[a_{2} \pm \sqrt{a_{2}^{2}-4 a_{4}\left(x-a_{2} t-a_{4} t^{2}\right)-4 \alpha_{7}}\right]
\end{aligned}
$$

$\alpha_{7}$ being an arbitrary constant of integration and $c_{3}=1 / e_{3}^{2}$, whereas $c_{1}$ and $c_{2}$ are linked by the relations $1-c_{1}^{2} e_{3}^{2}=0$ and $c_{2} e_{3}-a_{2}\left(1+c_{1} e_{3}\right)=0$.

A second solution can be obtained on the basis of the infinitesimal operator $\tilde{X}_{3}$ coupled along with the condition $c_{2}=0$; without loss of generality and in order to simplify the calculations, we neglect space and time translations.

Then the similarity variable and similarity solution read as

$$
\begin{aligned}
& z=\frac{x}{t^{\frac{1}{3}}}, \\
& u(x, t)=\frac{U(z)}{t^{\frac{2}{3}}}+\frac{V(z)}{t^{2}}, \quad v(x, t)=\frac{1-e_{2}}{2 e_{1}+e_{2}}\left(\frac{e_{2}}{e_{1}} \frac{U(z)}{t^{\frac{2}{3}}}+\frac{V(z)}{t^{2}}\right) .
\end{aligned}
$$

The reduced system of (1) assumes the form

$$
\begin{aligned}
& 3 e_{1} V^{\prime \prime \prime}+3 \frac{\left(e_{1}-e_{2}\right)\left(e_{2}-1\right)\left(2 e_{1}+e_{2}^{2}\right)}{\left(2 e_{1}+e_{2}\right)^{2}}\left[V^{\prime} U+U^{\prime} V\right]-e_{1} z V^{\prime}-6 e_{1} V=0, \\
& 3 e_{1} U^{\prime \prime \prime}+U^{\prime}\left[6 \frac{\left(e_{1}-e_{2}\right)\left(e_{2}-1\right)\left(e_{1}+e_{2}+e_{1} e_{2}\right)}{\left(2 e_{1}+e_{2}\right)^{2}} U-e_{1} z\right]-2 e_{1} U=0 .
\end{aligned}
$$

An exact solution of (18) is given by

$$
U(z)=\frac{e_{1}\left(2 e_{1}+e_{2}\right)}{2\left(e_{1}-e_{2}\right)\left(e_{2}-1\right)\left(e_{1}+e_{2}+e_{1} e_{2}\right)} z, \quad V(z)=0,
$$

where $e_{1}+e_{2}+e_{1} e_{2} \neq 0$ for compatibility with constraint (7).

Then, in terms of the original variables, a closed form solution of (1) is

$$
\begin{aligned}
& u(x, t)=\frac{e_{1}\left(2 e_{1}+e_{2}\right)}{2\left(e_{1}-e_{2}\right)\left(e_{2}-1\right)\left(e_{1}+e_{2}+e_{1} e_{2}\right)} \frac{x}{t}, \\
& v(x, t)=\frac{e_{2}}{2\left(e_{2}-e_{1}\right)\left(e_{1}+e_{2}+e_{1} e_{2}\right)} \frac{x}{t} .
\end{aligned}
$$

Of course, we may include time and space translations directly in the solution simply by means of the replacements $t \rightarrow t-t_{0}$ and $x \rightarrow x-x_{0}$, where $t_{0}$ and $x_{0}$ are arbitrary constants.

Two more similarity solutions for generalized coupled KdV equations (1) can be obtained from operator (5). Neglecting once again space and time translations, we obtain

$$
\begin{aligned}
& z=\frac{x}{t^{\frac{1}{3}}}-\frac{c_{2}\left(4 e_{1}-e_{2}^{2}\right)}{k} t^{\frac{2}{3}}, \\
& u(x, t)=\frac{2 c_{2} e_{1}}{k}+\frac{U(z)}{t^{\frac{2}{3}}}, \quad v(x, t)=-\frac{c_{2} e_{2}}{k}+\frac{V(z)}{t^{\frac{2}{3}}} .
\end{aligned}
$$


Then the reduced system assumes the following form:

$$
\begin{aligned}
& 3 c_{3} e_{3} U^{\prime \prime \prime}+3 V^{\prime \prime \prime}+3 c_{3}\left(2 e_{2} U^{\prime}+e_{1} V^{\prime}\right) U+\left(3 e_{1} c_{3} U^{\prime}+6 V^{\prime}-2 c_{1}\right) V-c_{1} z V^{\prime}=0, \\
& 3 U^{\prime \prime \prime}+3 e_{3} V^{\prime \prime \prime}+6 e_{1} V V^{\prime}-U^{\prime}\left(z-3 e_{2} V\right)+U\left(3 e_{2} V^{\prime}+6 U^{\prime}-2\right)=0 .
\end{aligned}
$$

An exact solution of (22) is

$$
U(z)=\frac{\alpha_{1}}{z^{2}}, \quad V(z)=\frac{\alpha_{2}}{z^{2}},
$$

$\alpha_{1}, \alpha_{2}$ being constants of integration that have to satisfy the following constraints:

$$
\begin{aligned}
& \alpha_{2}\left(6+\alpha_{2}\right)+c_{3} \alpha_{1}\left(6 e_{3}+e_{2} \alpha_{1}+e_{1} \alpha_{2}\right)=0 \\
& \alpha_{2}\left(6 e_{3}+e_{1} \alpha_{2}\right)+\alpha_{1}\left(6+e_{2} \alpha_{2}\right)+\alpha_{1}^{2}=0 .
\end{aligned}
$$

Then the corresponding solution of (1) is

$$
\begin{aligned}
& u(x, t)=\frac{2 c_{2} e_{1}}{k}+\frac{k^{2} \alpha_{1}}{\left[c_{2}\left(e_{2}^{2}-4 e_{1}\right) t+k x\right]^{2}}, \\
& v(x, t)=-\frac{c_{2} e_{2}}{k}+\frac{k^{2} \alpha_{2}}{\left[c_{2}\left(e_{2}^{2}-4 e_{1}\right) t+k x\right]^{2}} .
\end{aligned}
$$

In particular, when $c_{3}=0$, excluding the trivial solutions for (24), we are able to write the solution (25) for $\alpha_{1}=3\left(-1+e_{2}+\sqrt{\left(e_{2}-1\right)^{2}+4 e_{3}-4 e_{1}}\right)$ and $\alpha_{2}=-6$.

In the special case $k=c_{2}=0$, if we consider the linear combination of operators $\dot{X}_{3}$ and $\dot{X}_{4},\left(a_{3} \dot{X}_{3}+a_{4} \dot{X}_{4}\right)$ the similarity variables, the reduced system and a possible solution are exactly (21), (24) and (25) respectively, where $k$ and $c_{2}$ are considered as coefficients of the linear combination of two operators $\left(k=a_{3}\right.$ and $\left.c_{2}=a_{4}\right)$.

Considering once again the infinitesimal operator (5), we have a second type of solutions that can be expressed in terms of remarkable functions. Skipping the details of calculations, in terms of the original variables, the corresponding solution of (1) reads as

$$
\begin{aligned}
u(x, t)= & \frac{2 c_{2} e_{1}}{k}+\left[\frac{x}{t}-\frac{c_{2}\left(4 e_{1}-e_{2}^{2}\right)}{k}\right] \\
& \times\left[\frac{B\left(-\frac{2}{3}, 2 y\right) \Gamma\left(\frac{1}{3}\right) K_{1}+(-1)^{\frac{2}{3}} B\left(\frac{2}{3}, 2 y\right) \Gamma\left(\frac{5}{3}\right) K_{3}+(-1)^{\frac{1}{3}} H\left[\{1\},\left\{\frac{2}{3}, \frac{4}{3}\right\}, y^{2}\right] K_{2}}{3\left(1+e_{3} \lambda\right)^{\frac{1}{3}}}\right], \\
v(x, t)= & -\frac{c_{2} e_{2}}{k}+\lambda\left[\frac{x}{t}-\frac{c_{2}\left(4 e_{1}-e_{2}^{2}\right)}{k}\right] \\
& \times\left[\frac{B\left(-\frac{2}{3}, 2 y\right) \Gamma\left(\frac{1}{3}\right) K_{1}+(-1)^{\frac{2}{3}} B\left(\frac{2}{3}, 2 y\right) \Gamma\left(\frac{5}{3}\right) K_{3}+(-1)^{\frac{1}{3}} H\left[\{1\},\left\{\frac{2}{3}, \frac{4}{3}\right\}, y^{2}\right] K_{2}}{3\left(1+e_{3} \lambda\right)^{\frac{1}{3}}}\right],
\end{aligned}
$$

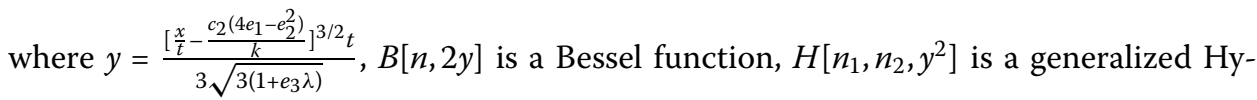
pergeometric function, while $\Gamma(\cdot)$ represents an Euler gamma function, and $K_{i}(i=1,2,3)$ constants of integration. 

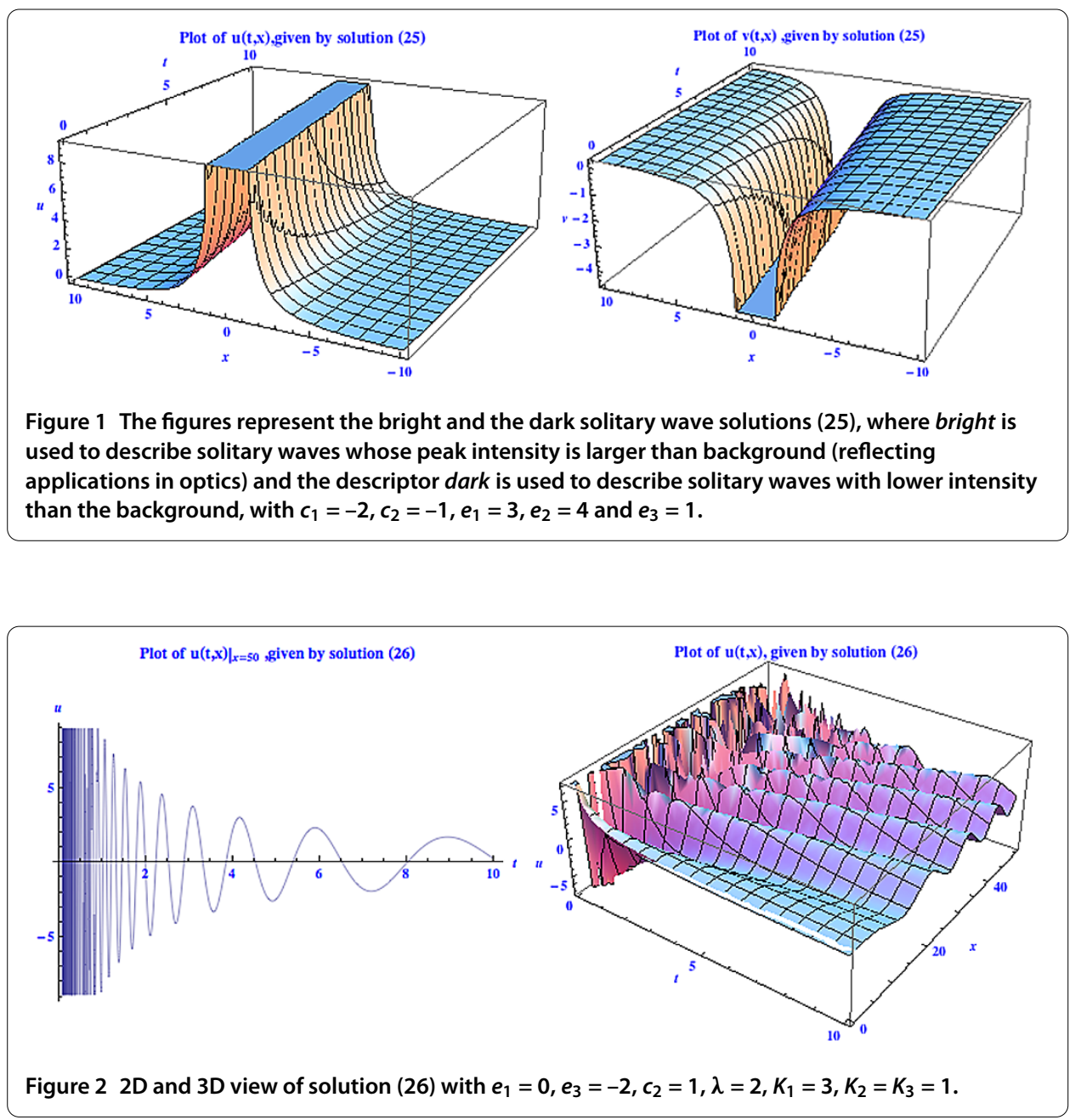

Moreover, $c_{2}, e_{3}, \lambda$ are arbitrary constants, $c_{1}=\frac{1+e_{3} \lambda^{2}}{1+e_{3} \lambda}, c_{3}=\lambda^{3}, e_{1}$ and $e_{2}$ are linked by the relation $\lambda^{2} e_{1}+\lambda e_{2}+1=0$ (for instance, if $e_{1}=0$, then $e_{2}=-\frac{1}{\lambda}$; on the contrary, $e_{2}=0$ implies $\left.e_{1}=-\frac{1}{\lambda^{2}}\right)$.

Finally, another solution of (1) can be obtained by using a linear combination of the operators $X_{1}, X_{2}$ and $X_{3}$, i.e., considering the operator $X_{1}+a_{2} X_{2}+a_{3} X_{3}$ along with the condition $k=0$.

Then the similarity variable and similarity solution are

$$
\begin{aligned}
& z=x-a_{2} t-a_{3}\left(4 e_{1}-e_{2}^{2}\right) t^{2}, \\
& u(x, t)=2 e_{1} a_{3} t+U(z), \quad v(x, t)=-e_{2} a_{3} t+V(z),
\end{aligned}
$$

respectively.

The reduced system of (1) assumes the form

$$
\begin{aligned}
& U^{\prime \prime \prime}+3 V^{\prime \prime \prime}+U^{\prime}\left(e_{2} V+2 U-a_{2}\right)+V^{\prime}\left(2 e_{1} V+e_{2} U\right)+2 a_{3} e_{1}=0, \\
& c_{3} e_{3} U^{\prime \prime \prime}+V^{\prime \prime \prime}+c_{3} U^{\prime}\left(2 e_{2} U+e_{1} V\right)+V^{\prime}\left(c_{3} e_{1} U+2 V-a_{2} c_{1}+c_{2}\right)-a_{3} c_{1} e_{2}=0 .
\end{aligned}
$$




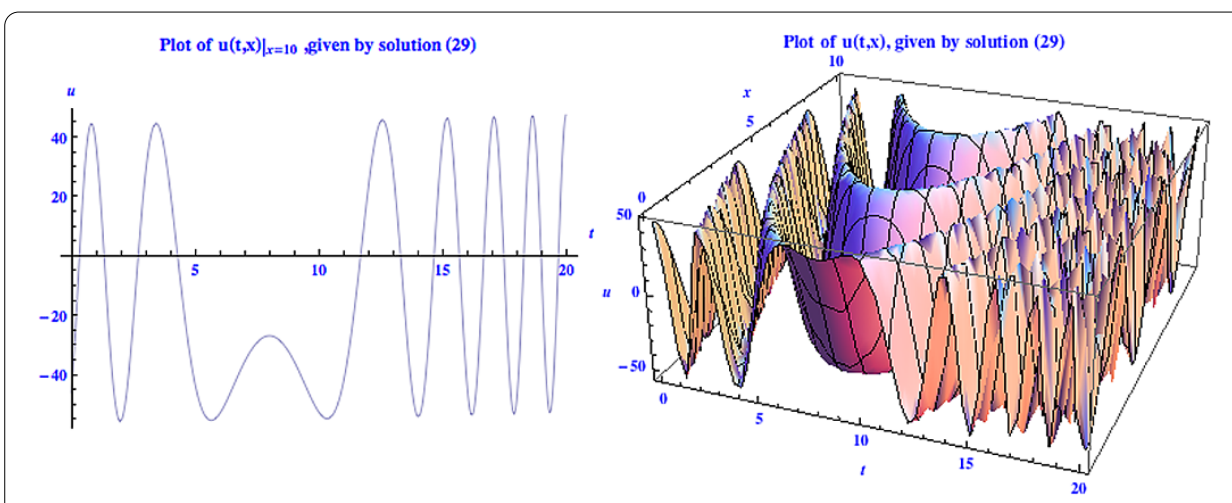

Figure $32 \mathrm{D}$ and $3 \mathrm{D}$ view of solution (29) with $e_{2}=0, \lambda=4, a_{2}=-2, a_{3}=-1, \alpha_{6}=10$ and $K_{1}=K_{2}=25$.

Then, in terms of the original variables, a closed form solution of (1) reads as

$$
\begin{aligned}
& u(x, t)=2 e_{1} a_{3} t+\frac{-2 a_{3} z+\alpha_{6} \lambda^{2}}{\lambda^{2} a_{2}}+K_{1} e^{\sqrt{\frac{a_{2}(\lambda-1)}{\lambda}} z}+K_{2} e^{-\sqrt{\frac{a_{2}(\lambda-1)}{\lambda}} z,} \\
& v(x, t)=-e_{2} a_{3} t+\lambda \frac{-2 a_{3} z+\alpha_{6} \lambda^{2}}{\lambda^{2} a_{2}}+K_{1} e^{\sqrt{\frac{a_{2}(\lambda-1)}{\lambda}}} z+K_{2} e^{-\sqrt{\frac{a_{2}(\lambda-1)}{\lambda}} z},
\end{aligned}
$$

with $z=x-a_{2} t-a_{3}\left(4 e_{1}-e_{2}^{2}\right) t^{2}$, while $\lambda, e_{1}$ and $e_{2}$ are linked by the relation $\lambda^{2} e_{1}+\lambda e_{2}+1=0$, $\alpha_{6}$ being an arbitrary constant of integration.

In this case, space and time translation must be included to obtain a relevant new similarity variable.

\subsection{Analysis of solutions}

The solutions we have found, apart from their own theoretical value, can be used as a benchmark test for numerical schemes and codes.

In particular, by the analysis of the solutions obtained which are invariant with respect to the symmetries of Case 1, we can observe that, as shown in Figure 1, the behavior of solution (25) is governed by the Bessel function which is decreasing with increasing argument. Taking into consideration solution (26), we can observe, from Figure 2, an oscillatory profile of the obtained solution. Finally, for what concerns solution (29), we can observe that it can be counted among the rational type solutions as in [25].

In order to show the trend of some of the obtained solutions, just as an example, snapshots of solutions (25), (26) and (29) are shown in Figures 1-3.

Competing interests

The authors declare that they have no competing interests.

\section{Authors' contributions}

The authors wrote this paper in collaboration and with the same responsibility. All authors read and approved the final version of the manuscript.

\section{Author details}

${ }^{1}$ Faculty of Engineering and Architecture, Kore University of Enna, Enna, Italy. ${ }^{2}$ Department of Mathematics and Computer Science, University of Messina, Messina, Italy.

\section{Acknowledgements}

The authors acknowledge the financial support by G.N.F.M. of INdAM through the project Metodologie di tipo analitico e numerico per lo studio di problemi iperbolici ed iperbolico-parabolici di natura ondosa. 


\section{References}

1. Gear, JA, Grimshaw, R: Weak and strong interactions between internal solitary waves. Stud. Appl. Math. 70(3), 235-258 (1984)

2. Bona, JL, Ponce, G, Saut, J-C, Tom, M: A model system for strong interaction between internal solitary waves. Commun. Math. Phys. 143, 287-313 (1992)

3. Ruggieri, M, Valenti, A: Approximate symmetries in nonlinear viscoelastic media. Bound. Value Probl. 2013, Article ID 143 (2013). doi:10.1186/1687-2770-2013-143

4. Ruggieri, M, Speciale, MP: Approximate analysis of a generalized KdV-like equations (2013, to appear)

5. Olver, PJ: Applications of Lie Groups to Differential Equations. Springer, New York (1986)

6. Ibragimov, NH: Lie Group Analysis of Differential Equations. CRC Handbook. CRC Press, Boca Raton (1994)

7. Fushchych, WI, Shtelen, WM: Symmetry Analysis and Exact Solutions of Nonlinear Equations of Mathematical Physics. Kluwer Academic, Dordrecht (1993)

8. Ruggieri, M, Valenti, A: Symmetries and reduction techniques for dissipative models. J. Math. Phys. 50, 063506 (2009)

9. Ruggieri, M, Valenti, A: Exact solutions for a nonlinear model of dissipative media. J. Math. Phys. 52, 043520 (2011)

10. Ruggieri, M: Kink solutions for class of generalized dissipative equations. Abstr. Appl. Anal., 2012, Article ID 237135 (2012). doi:10.1155/2012/237135

11. Oliveri, F, Speciale, MP: Exact solutions to the equations of ideal gas-dynamics by means of the substitution principle. Int. J. Non-Linear Mech. 33, 585-592 (1998)

12. Oliveri, F, Speciale, MP: Exact solutions to the equations of perfect gases through Lie group analysis and substitution principles. Int. J. Non-Linear Mech. 34, 1077-1087 (1999)

13. Oliveri, F, Speciale, MP: Exact solutions to the unsteady equations of perfect gases through Lie group analysis and substitution principles. Int. J. Non-Linear Mech. 37, 257-274 (2002)

14. Oliveri, F, Speciale, MP: Exact solutions to the ideal magneto-gas-dynamics equations through Lie group analysis and substitution principles. J. Phys. A, Math. Gen. 38, 8803-8820 (2005)

15. Margheriti, L, Speciale, MP: Unsteady solutions of Euler equations generated by steady solutions. Acta Appl. Math. 113, 289-303 (2011). doi:10.1007/s10440-010-9600-8

16. Donato, A, Oliveri, F: Reduction to autonomous form by group analysis and exact solutions of axi-symmetric MHD equations. Math. Comput. Model. 18, 83-90 (1993)

17. Donato, A, Oliveri, F: Linearization procedure of nonlinear first order systems of PDE's by means of canonical variables related to Lie groups of point transformations. J. Math. Anal. Appl. 188, 552-568 (1994)

18. Currò, C, Oliveri, F: Reduction of nonhomogeneous quasilinear $2 \times 2$ systems to homogeneous and autonomous form. J. Math. Phys. 49, 103504 (2008)

19. Oliveri, F: Lie symmetries of differential equations: classical results and recent contributions. Symmetry 2, 658-706 (2010)

20. Oliveri, F: General dynamical systems described by first order quasilinear PDEs reducible to homogeneous and autonomous form. Int. J. Non-Linear Mech. 47, 53-60 (2012)

21. Ovsiannikov, LV: Group Analysis of Differential Equations. Academic Press, New York (1982)

22. Lisle, IG: Equivalence transformations for classes of differential equations. PhD dissertation, University of British Columbia, Vancouver B.C., Canada (1992)

23. Oliveri, F, Speciale, MP: Equivalence transformations of quasilinear first order systems and reduction to autonomous and homogeneous form. Acta Appl. Math. 122, 447-460 (2012)

24. Oliveri, F, Speciale, MP: Reduction of balance laws to conservation laws by means of equivalence transformations. J. Math. Phys. 54, 041506 (2013)

25. Darwish, A, Fan, EG: A series of new explicit exact solutions for the coupled Klein-Gordon-Schrodinger equations Chaos Solitons Fractals 20, 609-617 (2004)

\section{Submit your manuscript to a SpringerOpen ${ }^{\ominus}$ journal and benefit from:}

- Convenient online submission

- Rigorous peer review

- Immediate publication on acceptance

- Open access: articles freely available online

- High visibility within the field

- Retaining the copyright to your article 